\title{
Anreize zur Nutzung von Wikis in der Hochschullehre: Ergebnisse eines Pilotprojekts
}

\author{
Stefan Seifert \\ Karlsruher Institut für Technologie \\ Institut für Informationswirtschaft und -management (IISM) \\ Jan Krämer \\ Karlsruher Institut für Technologie \\ Institut für Informationswirtschaft und -management (IISM)
}

Athanasios Mazarakis

FZI Forschungszentrum Informatik

\section{Zusammenfassung}

In einer Pilotstudie an der Universität Karlsruhe wurden Wiki-Systeme in die Hochschullehre integriert, mit dem Ziel die Studierenden schon während des Semesters zu einer aktiven Auseinandersetzung mit dem Lehrstoff zu animieren. In dieser Arbeit werden die wesentlichen Erkenntnisse der Studie vorgestellt, insbesondere im Hinblick auf die Auswirkungen verschiedener Nutzungsanreize auf die studentische Beteiligung in vorlesungsbegleitenden Wikis. In der Pilotstudie mit drei unterschiedlichen Lehrveranstaltungen lassen sich beachtliche Unterschiede hinsichtlich des studentischen Engagements aufgrund unterschiedlicher Anreizstrukturen beobachten. Es wird ebenso diskutiert, inwieweit sich die Erkenntnisse der Studie auf andere Lehrveranstaltungen übertragen lassen und welche Potenziale für die Hochschullehre damit verbunden sind. 


\section{$1 \quad$ Einleitung}

Studierende befinden sich hinsichtlich ihres Lern-Engagements in einem Dilemma: Einerseits akkreditieren sie ihren Kommilitonen schlechte Mitarbeit, andererseits lassen sie sich von deren „Trägheit“ anstecken und sind selbst nicht bereit, jenseits von Vorlesung oder Übung weitere Zeit in Vor- und Nachbereitung der Lehrveranstaltungen zu investieren.31 Diese Erkenntnis war Motivation für das Projekt „Interaktive Gestaltung von Lehrveranstaltungen mit Informationstechnologie (IGeL)“ an der Universität Karlsruhe. Ziel des Projekts war es, die Studierenden während des Semesters durch den Einsatz von Informationstechnologie kontinuierlich zu motivieren, sich mit den Inhalten der Vorlesung zu beschäftigen. Dieser Beitrag beschreibt die Erfahrungen und Erkenntnisse, die im Rahmen des Projektes gewonnen wurden, insbesondere in Bezug auf die Frage, ob und wie Studierende motiviert werden können, sich an einem vorlesungsbegleitenden Wiki zu beteiligen.

\section{$2 \quad$ Wikis in der Hochschullehre}

Gabriel, Gersch \& Weber (2008) betonen die Vorteile des sogenannten Blended LearningKonzepts, also der Verknüpfung von traditionellen Lehrelementen und E-Learning Komponenten, im Hinblick auf Flexibilitätssteigerung durch Orts- und Zeitunabhägigkeit, motivationale Effekte unter Berücksichtigung veränderter Medienerfahrungen, sowie vielseitige Individualisierungsmöglichkeiten unter Bezugnahme auf moderne lerntheoretische Ansätze. Hierfür ist der Einsatz eines vorlesungsbegleitenden Wikis grundsätzlich gut geeignet: Wikis unterstützen die gemeinschaftliche Arbeit an Dokumenten, indem sie allen Nutzern schreibenden Zugriff auf zentral gehaltene Dokumente gewähren und sämtliche Änderungen allen anderen Nutzern in Echtzeit zur Verfügung stellen (Cress \& Kimmerle, 2008, S. 106). Eventuelle Fehler können so schnell erkannt und einfach durch die Studierenden selbst oder den Dozenten korrigiert werden. Dadurch kann die aktive Beteiligung der Studierenden sowie die Interaktion mit dem Dozenten gefördert und gleichzeitig ein Skript erstellt werden, welches den Studierenden als zusätzliche Lernhilfe zur Verfügung steht. Darüber hinaus erlauben Wikis auch die Einbindung multimedialer Inhalte und verbessern dadurch im Vergleich zu traditionellen, gedruckten Skripten die multi-modale Wissensvermittlung (Weidemann, 2002, S. 53).

Die Grundgedanken von Wikis können nach Ebner (2007) unter folgenden drei Aspekten zusammengefasst werden: (i) Offenheit (Struktur und Inhalt ist theoretisch von jedem Teilnehmer gestaltbar), (ii) Beobachtbarkeit (der Inhalt ist protokollierbar), sowie (iii) eine organische Struktur (der Inhalt wächst und ist veränderbar).

Der wahrscheinlich größte Vorteil im Hochschulbereich liegt jedoch in der unmittelbaren Erstellung und Kommentierung von Texten durch die Studierenden und deren Nachverfol-

31 Dies ergab die Auswertung der wirtschaftswissenschaftlichen Lehrveranstaltungen an der Universität Karlsruhe im Wintersemester 2007/2008 (Studiendekanat der Fakultät für Wirtschaftswissenschaften, 2008). 
gung durch den Dozenten. Das Wiki-System bietet hierbei die Möglichkeit, korrigierend, ergänzend und kommentierend auf die von anderen eingestellten Beiträge zu reagieren. Somit kann einerseits eine Kontrolle der Inhalte durch das Mehr-Augen-Prinzip vorgenommen und die Kooperation gefördert werden (Moskaliuk \& Kimmerle, 2008, S. 3), anderseits erhält der Dozent bereits in der Erstellungsphase unmittelbare Informationen über den konkreten Lernerfolg und das bisherige Verständnis der Studierenden (Bruder \& Sonnberger, 2008, S. 236). Hiermit werden konträre Positionen deutlich, welche diskutiert werden können und gleichzeitig kann diese Form der Rückmeldung auch einen motivierenden Charakter für die Gruppenteilnehmer haben (Hesse, Garsoffky \& Hron, 2002, S. 287). Zusätzlich ist die Mitarbeit an einem Wiki eine soziale Tätigkeit, da kooperativ und kollektiv gemeinsam eine Aufgabe erledigt wird, welche zusätzlich durch den Besuch der gemeinsamen Lehrveranstaltung situiert ist (Moskaliuk \& Kimmerle, 2008, S. 15). Wikis werden hierbei nach Parker \& Chao (2007, S. 58) immer öfter in unterschiedlichen Szenarien an Hochschulen eingesetzt. Hervorgehoben wird die relativ einfache Installation der Software sowie die einfache Nutzung, die die Aufmerksamkeit auf Inhalte lenkt, anstatt mit technischen Details zu verwirren. Auch aus Sicht der Lehrenden sind diese Aspekte relevant, zumal Katzlinger \& Kepler (2008, S. 85) auch allgemein Wikis als Bereicherung in der universitären Lehre hervorheben. Eine ähnliche Einschätzung vertreten Kimmerle, Moskaliuk \& Cress (2009, S. 1), die die Haupteigenschaften beim Lernen mit Wikis in (i) der Gemeinschaft der Lernenden, (ii) der Nutzung eines gemeinsamen digitalen Artefakts zur kollaborativen Zusammenarbeit, (iii) den selbstregulierten Lernprozessen in informellen Settings, (iv) der Betonung des kollaborativen Charakters durch das gemeinsam erstellte Artefakt und (v) der Erwerbung neuer Expertise, über welche die Gemeinschaft vorher nicht verfügte, sehen.

Der Einsatz von Wikis in der Lehre wird in zahlreichen Projekten praktiziert und in der Literatur bereits auch vielfach diskutiert (z. B. Appelrath, 2009, S. S. 11). Allerdings untersuchen diese Studien nicht die konkrete Auswirkung von Anreizmechanismen zur Steigerung von Beteiligung und inhaltlicher Qualität. Vor diesem Hintergrund wurde im Wintersemester 2008/2009 eine Pilotstudie (,IGeL“) mit drei Veranstaltungen aus dem allgemeinen Curriculum der wirtschaftswissenschaftlichen Fakultät der Universität Karlsruhe (TH) durchgeführt. Neben der grundsätzlichen Praktikabilität von Wikis im Allgemeinen sowie der Eignung unterschiedlicher Wiki-Engines im Speziellen standen in Hinblick auf die obigen Projektziele insbesondere die folgenden Fragen im Vordergrund: (i) Fördern vorlesungsbegleitende Wikis die aktive Auseinandersetzung der Studierenden mit den Lehrinhalten? (ii) Sind für eine rege Beteiligung am Vorlesungswiki explizite, extrinsische Anreize notwendig und welche Anreizmechanismen eigenen sich ggf. im Besonderen? (iii) Wie ist Qualität der Inhalte zu bewerten, die von den Studierenden ins Wiki eingestellt wurden?

\section{$3 \quad$ Anreizmechanismen in der Pilotstudie}

Die drei für die Pilotstudie ausgewählten Lehrveranstaltungen weisen verschiedene Merkmale auf. (i) Die Vorlesung Management of Business Networks (MBN) ist eine englischsprachige Vorlesung, die auf strategische Managemententscheidungen abzielt. Neben Studierenden im Hauptstudium (Diplom/Master) wird die Vorlesung zu etwa gleichen Teilen auch von 
Bachelorstudenten besucht. Zentrales Medium der Vorlesung sind Powerpoint-Folien. Die Vorlesung wird dennoch sehr interaktiv abgehalten, nicht zuletzt da die studentische Mitarbeit mit $15 \%$ in die Gesamtnote einfließt. (ii) Die Vorlesung Grundzüge der Informationswirtschaft (GZI) ist eine Einführungsveranstaltung in das Gebiet der Informationswirtschaft und wird üblicherweise zu Beginn des Hauptstudiums gehört. Sie ist für Studierende der Informationswirtschaft obligatorisch und damit die einzige Pflichtveranstaltung der Studie. Zur Vermittlung der Vorlesungsinhalte werden überwiegend Powerpoint-Medien eingesetzt. (iii) Im Unterschied zur MBN und der GZI ist die Vorlesung Auktionstheorie (AT) eher mathematisch ausgerichtet. Sie richtet sich vorwiegend an Wirtschaftsingenieure, Informationswirte sowie Wirtschaftsmathematiker und wird in der Regel erst gegen Ende des Hauptstudiums gehört. Aufgrund ihrer formalen Prägung werden viele Inhalte an der Tafel entwickelt und Folien nur ergänzend eingesetzt.

In allen drei Veranstaltungen wurden die Studierenden aufgefordert, die einzelnen Vorlesungseinheiten auf Basis der vorhandenen Vorlesungsmaterialien (Folien, Tafelanschrieb) sowie des eigenen Vorlesungsmitschriebs im Wiki zu dokumentieren. Auf diese Weise sollte im Lauf des Semesters ein kollaborativ erstelltes Vorlesungsskript entstehen. In dem Semester, in dem die Pilotstudie durchgeführt wurde, gab es in der MBN und der GZI noch keine Texte im Wiki, auf denen die Studierenden ihre Beiträge hätten aufbauen können. Lediglich für die Auktionstheorie lagen bereits zu Semesterbeginn einzelne Kapitel vor, die von den Dozenten in das Wiki eingepflegt wurden.

Neben den oben genannten Punkten unterscheiden sich die drei Veranstaltungen insbesondere auch in Hinblick auf die Anreize zur Mitwirkung. Bereits erwähnt wurde, dass in der MBN die Mitarbeit bewertet und in die Gesamtnote eingerechnet wurde. Für die Beteiligung am Vorlesungswiki wurde kein expliziter zusätzlicher Anreiz gesetzt, sondern darauf hingewiesen, dass die Erstellung sowie die Bearbeitung und Ergänzung von Vorlesungsprotokollen positiv in die Mitarbeitsnote einfließen. Weitere Anreize oder organisatorische Hilfestellungen bspw. durch eine Einteilung in Arbeitsgruppen wurden jedoch nicht gegeben. Von daher kann diese Vorlesung als Kontrollgruppe betrachten werden.

In der GZI konnten die Studierenden durch die aktive Beteiligung am Wiki einen Punktebonus erhalten, der zu den in der Abschlussklausur erzielten Punkten addiert wurde. ${ }^{32}$ Hierzu mussten sie sich zunächst selbständig in Gruppen von bis zu vier Studierenden einteilen. Jeder Gruppe wurde ein Vorlesungstermin zugewiesen, zu dem innerhalb einer Woche ein Vorlesungsprotokoll anzulegen war. Wurde das Protokoll rechtzeitig erstellt, so erhielt jedes Gruppenmitglied einen Bonus von zwei Verrechnungspunkten auf die Klausur. Zusätzlich zu diesem Gruppenbonus konnte jeder Studierende einen Individualbonus von maximal zwei Punkten erreichen, wenn er sich aktiv an der Inhaltskontrolle der anderen Protokolle beteiligte und diese entsprechend verbesserte und erweiterte. Über die Versionskontrolle des Wikis konnte dies individuell erfasst werden. Damit waren für die Mitarbeit am Wiki insgesamt bis

\footnotetext{
32 Neben dem Bonus für die Mitarbeit am Wiki konnten die Studenten auch durch die regelmäßige Abgabe von Übungsaufgaben und Teilnahme an der Übung Bonuspunkte für die Klausur erhalten. Die beiden Boni waren unabhängig voneinander und wurden addiert.
} 
zu vier Punkte erreichbar, was einer Verbesserung der Gesamtnote um einen Schritt von einer Drittelnote $(0,3)$ entspricht.

Ein ähnlicher Mechanismus wurde für die AT gewählt. Hier war die Gruppengröße jedoch auf zwei Studierende beschränkt. Im AT-Wiki wurde bereits zu Beginn des Semesters eine Gliederung der Vorlesung mit den einzelnen Inhalten zu den jeweiligen Vorlesungsterminen ins Wiki eingestellt und die Studierenden konnten sich selbst zu den Terminen als Protokollanten eintragen. Für die initiale Erstellung eines Protokolls wurden zwei Punkte (an beide Mitglieder der Gruppe) vergeben. Bis zu drei weitere (individuelle) Punkte konnten durch die Überarbeitung anderer Protokolle bzw. die Übertragung von Inhalten ins Skript und dessen Verbesserung verdient werden. Auch hier entsprach der insgesamt erreichbare Bonus einer Verbesserung der Klausurnote um einen Schritt von einer Drittelnote $(0,3)$. Außerdem wurde in der AT-Veranstaltung mit den Studierenden das Ergebnis der letzten Lehrevaluation besprochen und ausdrücklich das Ziel vereinbart, im Laufe des Semesters gemeinsam ein Vorlesungsskript zu erstellen. Die Vereinbarung eines solchen gemeinsamen Ziels folgt der Zielsetzungstheorie nach Locke (2001, S. 45). Da sie direkt dem Bedürfnis der Vorjahresstudenten folgte, sollte sie zu einer hohen Zielidentifikation und -akzeptanz führen und damit die Mitarbeit in besonderem Maße fördern (ebd., S. 46). Die Wichtigkeit von (impliziten) Zielen bei der Arbeit mit Wikis wird auch von Moskaliuk \& Kimmerle (2008, S. 10) bestätigt. Zusätzlich wird dadurch ein üblicher Schwachpunkt nach Hugl (2010, S. 218) umgangen: Das Lernziel ist aus der eigenen peer-group entstanden und nicht aus dem Lehrveranstaltungskalender übernommen, was für das angenommene konstruktivistische Lernparadigma bei Wikis von Vorteil ist. Vom Brocke, Grob, Buddendick \& Simons (2010, S. 34) sehen in der organisationspsychologischen Zielsetzungstheorie von Locke die Möglichkeit, übergeordnete Ziele wie die Erstellung des Skripts, auf die individuellen Bedürfnisse auszurichten, nämlich durch einen auf die Situation passenden Anreiz, in diesem Fall einem Klausurbonus. Im Gegensatz zum eher abstrakten Aufzeigen von Anreizen, wurden in der vorliegenden Pilotstudie daher explizite Anreize gesetzt.

Explizite Anreize wurden auch bei anderen Studien eingesetzt. Während Katzlinger \& Kepler (2008) keine expliziten Anreizen bei der Erstellung von Beiträgen im Wiki nennen und nur von einer pauschalen Leistungsbeurteilung sprechen, versuchen Hermann \& Janzen (2009), mit einem materiellen Anreiz, dem Gewinn eines USB-Sticks für den besten Artikel, die Beteiligung zu erhöhen. Die Beteiligung nahm in der Studie von Hermann \& Janzen nach Wegnahme des Anreizes ab und es wurde ähnlich wie bei der Evaluation durch das Studiendekanat der Universität Karlsruhe (2008, S.11) die fehlende Motivation der Kommilitonen bemängelt.

Auch die alleinige Betonung von intrinsischer Motivation im Rahmen der Selbstbestimmungstheorie von Deci \& Ryan (2000) mit den drei psychologischen Grundbedürfnissen Autonomie, Kompetenz und sozialer Eingebundenheit kann als Konstrukt zur Steigerung der Partizipation verstanden werden. Moskaliuk \& Kimmerle (2008, S. 11) verweisen darauf, dass die Beteiligung an einem Wiki das Potenzial besitzt diese drei Grundbedürfnisse zu befriedigen. In der vorliegenden Pilotstudie wurden jedoch zusätzlich explizite, extrinsische Anreize verwendet und auf ihre grundsätzliche Verwendbarkeit bei Vorlesungswikis hin untersucht. 
Zur Evaluation der unterschiedlichen Anreizmechanismen und des Einsatzes von Wikis in der Hochschullehre wurde in der GZI und der AT per Fragebogen etwa zur Mitte des Semesters eine Umfrage zur Motivation der Studierenden bzgl. der Mitarbeit am Vorlesungswiki, dem zeitlichen Aufwand sowie der generellen Akzeptanz eines Vorlesungswikis durchgeführt. Mit den Studenten der GZI wurde die Umfrage nach Ende des Semesters und nach der Klausur, aber vor Bekanntgabe der Klausurergebnisse als Online-Befragung wiederholt. Auf einige ausgewählte Ergebnisse der Befragung wird im Folgenden näher eingegangen.

\section{$4 \quad$ Ergebnisse und Diskussion}

Festzuhalten ist zunächst, dass von den 30 Studierenden der MBN über das gesamte Semester kein einziger Eintrag im Wiki notiert wurde. Dieses Ergebnis ist identisch mit den Erfahrungen von Ebner, Kickmeier-Rust \& Holzinger (2008, S. 205).

In der GZI konstituierten sich zwölf studentische Gruppen mit insgesamt 45 Studierenden. Damit nahmen 90\% der 50 Studierenden, die im Anschluss an die Vorlesung geprüft wurden, die Möglichkeit wahr, sich durch die Mitarbeit am Wiki einen Klausurbonus zu verdienen. Für sechs der initialen Vorlesungsprotokolle wurde die Höchstzahl von zwei Punkten vergeben, viermal wurden 1,5 Punkte vergeben und zweimal ein Punkt.

In der AT beteiligten sich insgesamt 13 Studierende von 20 Studierenden am Wiki. Zwölf von ihnen legten insgesamt neun Protokolle an, für die jeweils der volle Bonus von zwei Punkten vergeben wurde. Ein weiterer Student beteiligte sich ausschließlich an der Überarbeitung anderer Beiträge. Entstanden ist ein Skript, das zum Semesterende 101 Seiten umfasste. Im Zuge der Klausurvorbereitung wuchs es auf 111 Seiten an und auch nach der (Haupt-)klausur wurde das Skript von den Studierenden weiter gepflegt. Inzwischen umfasst es insgesamt über 130 Seiten.

Auffällig ist, dass sich bei der AT im Vergleich zur GZI nur die Hälfte der Studierenden am Wiki beteiligte. Eine mögliche Ursache könnte darin liegen, dass aufgrund der kleineren Gruppengröße der Aufwand einer Beteiligung am Wiki als zu hoch eingeschätzt wurde. Ein Indiz dafür, dass der Aufwand für die Protokollerstellung in der AT tatsächlich höher war als in der GZI liefert die Umfrage unter den Studierenden. So gaben die Studierenden der GZI zur Semestermitte an, im Mittel 162 Minuten für die bisherige Protokollerstellung aufgewendet $\mathrm{zu}$ haben, bei den Studierenden der AT liegt dieser Wert bei 238 Minuten. ${ }^{33}$ Umgekehrt waren die Studierenden, die sich am AT-Wiki beteiligten, außerordentlich engagiert. Neben dem bereits genannten höheren zeitlichen Einsatz produzierten die Studierenden der AT mehr Text, verfassten mehr Beiträge und erhielten dafür auch pro Kopf mehr Bonuspunkte als ihre Kommilitonen in der GZI. Auch das formulierte Ziel der gemeinsamen Skripterstellung kann eine Rolle für das hohe Engagement gespielt haben. Dies wäre ein

33 Die Zahlenangaben sind nicht unproblematisch. Berücksichtigt wurden nur die Fragebögen der Studenten, die zum Zeitpunkt der Umfrage nach eigenen Angaben bereits ein Protokoll angelegt hatten; dies ist für weniger als die Hälfte der insgesamt aktiven Studierenden der Fall. Insgesamt liegen aus der GZI nur elf und aus der AT lediglich vier entsprechende Angaben vor. 
Beleg für die Anwendbarkeit der Zielsetzungstheorie von Locke (2001, S. 46). Die Tatsache, dass auch nach Festlegung und Bekanntgabe der Bonuspunkte weiter am Skript gearbeitet wurde, ist ein zusätzlicher Hinweis in diese Richtung.

Der Einsatz eines Wikis verfolgte in IGeL das Ziel, den Lernerfolg der Studierenden zu verbessern. Zur Überprüfung, inwieweit dieses Ziel erreicht wurde, wurden die Studierenden in der Umfrage gefragt, wie häufig sie das Wiki nutzen. Dabei wurde zwischen aktiver und passiver Nutzung unterschieden. Die Ergebnisse belegen eine hohe passive Nutzung. Insgesamt $80 \%$ der Studierenden verwendeten das Wiki mindestens einmal pro Monat. Wöchentlich wurde das Wiki in der GZI während des Semesters von 75\% der Studierenden passiv genutzt. In der AT liegt dieser Wert bei nur 36\%. Nach Ende des Semesters ging im Rahmen der Klausurvorbereitung auch bei der GZI die wöchentliche Nutzung auf $46 \%$ der Studierenden zurück. Bemerkenswert ist insbesondere die hohe Nutzung in der AT: Während sich nur etwa die Hälfte der Studierenden an der Erstellung von Beiträgen beteiligte, nutzten dennoch 79\% das Wiki mindestens einmal im Monat passiv. Nicht ganz erreicht wurde das Ziel der kontinuierlichen, vorlesungsbegleitenden aktiven Nutzung. Der Anteil der Studierenden, die das Wiki wenigstens einmal pro Monat aktiv nutzten, ist bei keiner der Umfragen größer als $50 \%$. Die wöchentliche Nutzung liegt bei der GZI sogar nur bei rund 10\%. Möglicherweise beschränkte sich die aktive Nutzung nur auf die Erarbeitung von Bonuspunkten im Rahmen von ein oder zwei Arbeitssitzungen im Laufe des Semesters. Ein größeres Engagement zeigten hier wiederum die Studierenden in der AT. Während sich weniger als die Hälfte der Studierenden überhaupt aktiv am Wiki beteiligten, lag der Anteil derer, die wenigsten einmal pro Woche aktiv waren, immerhin bei $20 \%$. Obwohl die Aktivität (besonders unter den Studierenden selbst) noch nicht die gesetzten Erwartungen erfüllt hat, so ist doch die aktive Teilnahme sichtbar und erfolgreicher, als zum Beispiel bei den zwei bekannten BauwikiUntersuchungen von Ebner (2007, S. 142).

Nicht erfüllt hat sich im Projekt die Hoffnung, dass über das Wiki intensive Diskussionen innerhalb der Gruppe der Studierenden sowie zwischen ihnen und den Dozenten entstehen würden. Wie bei Katzlinger \& Kepler (2008, S. 84) waren die Arbeiten eher Einzelarbeiten und die gemeinsame Arbeit mit anderen Studierenden war nicht stark ausgeprägt. Auffallend ist insbesondere die Scheu, Beiträge anderer Personen zu verändern. Rechtschreib- und Tippfehler wurden noch am ehesten korrigiert. Auch von der Möglichkeit, Inhalte untereinander zu verlinken und damit thematisch verwandte Beiträge schneller auswählen zu können, wurde ebenso wie bei Hugl (2010, S. 223) kaum Gebrauch gemacht.

Inwieweit das ursprüngliche Ziel, die Verbesserung des Lernerfolgs, erreicht wurde, ist nicht zweifelsfrei zu belegen. Es gibt hierfür jedoch zwei Indizien: Zum einen wurden sowohl in der GZI als auch in der AT bessere Leistungen als in den Vorjahren erzielt: selbst ohne Einrechnung der Boni lagen die Noten der Haupt- und Nachklausur der GZI sowie der Hauptklausur der AT im Schnitt um etwa eine Drittelnote besser als in den Vorjahren. Zum anderen schnitten die Studierenden, die sich aktiv am Wiki beteiligt hatten, besonders gut in der Klausur ab. Diese Erkenntnis muss jedoch keine Kausalität darstellen, sondern kann sich auch aus einer Korrelation mit weiteren Erklärungsmöglichkeiten ergeben: Es ist durchaus denkbar, dass sich insbesondere die ,besseren“ Studierenden mit dem Wiki beschäftigt haben. Denkbar ist auch, dass bestimmte Studierende stärker auf den angebotenen Anreiz rea- 
giert haben als Studierende, die weniger extrinsisch motiviert sind. Im ungünstigsten Fall könnte es zu einem Korrumpierungseffekt kommen, also dem Nachlassen von eigenständiger Motivation durch die Darbietung extrinsischer Anreize. Der Effekt wird jedoch nur in seltenen Situationen beobachtet (Deci, Koestner \& Ryan, 2001, S. 9), und in der vorliegenden Studie findet sich darauf kein Hinweis.

In der Pilotstudie sind aber auch beachtliche Unterschiede hinsichtlich des studentischen Engagements zwischen den einzelnen Vorlesungen und den jeweiligen Rahmenbedingungen zu erkennen. Vor dem Hintergrund der Beteiligung in der GZI und der AT fällt insbesondere die völlige Zurückhaltung in der MBN bei der Mitarbeit am Wiki auf. Es liegt die Vermutung nahe, dass diese mit dem Fehlen expliziter Anreize sowie der mangelnden organisatorischen Unterstützung zusammenhängt. Bemerkenswert ist, dass Anreize nicht generell fehlten; eine Beteiligung am Wiki hätte sich sehr wohl in der Mitarbeitsnote niedergeschlagen. Allerdings war das Verhältnis von Aufwand in Bezug auf eine Verbesserung der Note nicht bekannt. Insbesondere der Verfasser des ersten Eintrags kann kaum abschätzen, welche Leistung erwartet wird - er weiß nur, dass er alleine allenfalls einen kleinen Beitrag im Verhältnis zur gesamten Vorlesung erbringen kann. Von daher hatte die Zuteilung von Vorlesungsterminen zu Protokollgruppen mehrere Vorteile: Erstens war der geforderte Aufwand eines Protokolls beschränkt und im Vorfeld bekannt, zweitens wurde über das Punktesystem für jedes einzelne Protokoll eine klare Belohnung in Aussicht gestellt und drittens wurden über die initialen Protokolle sehr schnell Referenzgrößen und Anknüpfungspunkte für die Arbeit anderer geschaffen. Um die aktive, studentische Mitarbeit generell zu verbessern, erscheint es daher sinnvoll, explizite Anreize für überschaubare Arbeitspakete zu vergeben. Möglicherweise sind hierfür selbst einzelne Protokolle von ganzen Vorlesungseinheiten über 90 Minuten nicht filigran genug. Darüber hinaus wäre für zukünftige Anwendungen zu prüfen, wie die Anreize für die Überarbeitung anderer Beiträge zielgerichteter gestaltet werden können, um die Kontinuität der Mitarbeit weiter zu fördern. Interessant ist hierbei, dass nach Ebner et al. (2008, S. 201) die Setzung von Anreizen die metakognitiven Prozesse wie Selbstreflektion der eigenen Beiträge und die Reflektion von fremden Beiträgen negativ beeinflussen kann. Dies wird mit der Unvereinbarkeit des Wiki-Prinzips und extrinsischen Anreizen begründet. Zusätzlich betonen Ebner et al. (2008, S. 206), dass es sich hier eher um ein soziologisches oder psychologisches Problem handelt und weniger um ein technologisches. Aber vielleicht sind in diesem eng gesteckten Setting (kurze Zeitdauer von Vorlesungen/Seminaren; meistens muss am Ende noch eine individuelle Prüfungsleistung erbracht werden; fehlende Verknüpfung zwischen den einzelnen Vorlesungen/Seminaren an der Universität) genau solche Anreize notwendig, um die Studierenden zu einer weitergehenden Beschäftigung mit den Inhalten anzuhalten. Denn obwohl Ebner et al. (2008, S. 205) hierbei Gefahren im konstruktivistischen Lernprozess sehen, so muss doch die Vermittlung des Stoffes und die erfolgreiche Prüfungsleistung weiterhin im Vordergrund stehen.

Abschließend ist festzuhalten, dass weiterer Forschungsbedarf besteht, inwieweit sich durch die Fortführung der bisher erstellten Wikis Lerneffekte auf die kommenden Jahrgänge übertragen lassen (vgl. Bruder et al., 2008, S. 237) und ob signifikante Unterschiede in der Quantität und Qualität der Inhalte entstehen, nachdem die Initialphase der Pilotstudie abgeschlossen wurde. 


\section{Danksagung}

Wir danken den Studierenden Philipp Benzko und Tim Straub für ihre Mitarbeit und die Erhebung der Daten im Rahmen eines Seminars.

\section{Kontaktinformationen}

Athanasios Mazarakis (FZI Forschungszentrum Informatik)

Telefon: +49 (721) 9654-848

Telefax: +49 (721) 9654-849

E-Mail: mazarakis@fzi.de

\section{$5 \quad$ Literaturverzeichnis}

Appelrath, H. (2009). Mit ELAN E-Learning in den Hochschulen ausbauen. In: H. Appelrath \& L. Schulze (Hrsg.). Auf dem Weg zu exzellentem E-Learning: Vernetzung und Kooperation der Hochschullehre in Niedersachsen (S. 11-19). Münster: Waxmann.

Bruder, R. \& Sonnberger, J. (2006). Die Qualität steckt im Detail - kreative Aufgabengestaltung und ihre Umsetzung mit E-Learning-Lösungen. In: S. Zauchner, P. Baumgartner, E. Blaschitz \& A. Weissenbäck (Hrsg.). Offener Bildungsraum Hochschule - Medien in der Wissenschaft, 48 (S. 228238). Münster: Waxmann.

Cress, U. \& Kimmerle, J. (2008). A systemic and cognitive view on collaborative knowledge building with wikis. International Journal of Computer-Supported Collaborative Learning, 3(2), 105-122.

Deci, E. \& Ryan, R. (2000). The "what" and "why" of goal pursuits: Human needs and the selfdetermination of behavior. Psychological Inquiry, 11, 227-268.

Deci, E., Koestner, R. \& Ryan, R. (2001). Extrinsic Rewards and Intrinsic Motivation in Education: Reconsidered Once Again. Review of Educational Research, 71, 1-27.

Ebner, M. (2007). Wikipedia - Hype oder Zukunftshoffnung für die Hochschullehre. In: E-Learning: Strategische Implementierungen und Studieneingang, Tagungsband zur 13. FNMA-Tagung (S. 139-146). Graz: Forum Neue Medien Austria.

Ebner, M., Kickmeier-Rust, M. \& Holzinger, A. (2008). Utilizing Wiki-Systems in higher education classes: a chance for universal access? Universal Access in the Information Society, 7, 199-207.

Gabriel, R., Gersch, M. \& Weber, P. (2008). Möglichkeiten und Grenzen von Lern-Services. Wirtschaftswissenschaftliches Studium, 10, 563-565. 
Hermann, C. \& Janzen, A. (2009). Electures-Wiki - Aktive Nutzung von Vorlesungsaufzeichnungen. In: A. Schwill \& N. Apostolopoulos (Hrsg.). Lernen im Digitalen Zeitalter-DeLFI 2009 - Die 7. E-Learning Fachtagung Informatik (S. 127-138). Bonn: Köllen.

Hesse, F., Garsoffky, B. \& Hron, A. (2002). Netzbasiertes kooperatives Lernen. In: L. Issing \& P. Klimsa (Hrsg.), Information und Lernen mit Multimedia und Internet (S. 283-298). Weinheim: Beltz.

Hugl, U. (2010). Work in Progress: Wiki-Einsatz als Blended Learning-Instrument in der universitären Lehre. In: M. Breitner, F. Lehner, J. Staff \& U. Winand (Hrsg.), E-Learning 2010. Aspekte der Betriebswirtschaftslehre und Informatik (S. 215-228). Heidelberg: Springer.

Katzlinger, E. \& Kepler, J. (2008). Wikis als kollaborative Lernform in der universitären Lehre. In: ELearning: Innovative Didaktik in berufsbegleitenden und Vollzeit-Studiengängen, Tagungsband zur 17. FNMA-Tagung (S. 80-86). Graz: Forum Neue Medien Austria.

Kimmerle, J., Moskaliuk, J. \& Cress, U. (2009). Understanding Learning - the Wiki Way. In: Proceedings of the $5^{\text {th }}$ international Symposium on Wikis and Open Collaboration, WikiSym09 (S. 1-8). New York: ACM.

Locke, E. (2001). Motivation by Goal Setting. In: Golembiewski, R. (Hrsg.), Handbook of Organizational Behavior. 2. rev. and expanded ed. New York et al.: Dekker.

Moskaliuk, J. \& Kimmerle, J. (2008). Wikis in der Hochschule - Faktoren für den erfolgreichen Einsatz. Verfügbar unter http://www.e-teaching.org/didaktik/kommunikation/wikis/08-1119_Moskaliuk-Kimmmerle_Wikis.pdf [24.2.2010]

Parker, K. \& Chao, J. (2007). Wiki as a Teaching Tool. Interdisciplinary Journal of Knowledge and Learning Objects, 3, 57-72.

Studiendekanat der Fakultät für Wirtschaftswissenschaften, Universität Karlsruhe (TH) (2008), Entwurf eines Berichts über die studentische Lehrveranstaltungsevaluation, WS 2007/2008.

Vom Brocke, J., Grob, H., Buddendick, C. \& Simons, A. (2010). Anreizsysteme für die E-LearningIntegration: Entwicklung eines Vorgehensmodells für die leistungsorientierte Budgetierung an Hochschulen. In: M. Breitner, F. Lehner, J. Staff \& U. Winand (Hrsg.), E-Learning 2010. Aspekte der Betriebswirtschaftslehre und Informatik (S. 31-45). Heidelberg: Springer.

Weidemann, B. (2002). Multicodierung und Multimodalität im Lernprozess. In: L. Issing \& P. Klimsa (Hrsg.), Information und Lernen mit Multimedia und Internet (S. 45-62). Weinheim: Beltz. 